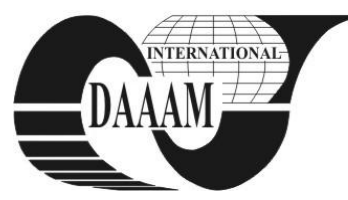

\title{
ROBUST FAULT DIAGNOSIS IN LINEAR SYSTEMS
}

\section{FILARETOV, V[ladimir] F[edorovich]; ZHIRABOK, A[lexey] N[il]; SHUMSKY, A[lexey] Y[evgeny] \& SUVOROV, A[lexey]}

\begin{abstract}
The problem of robust fault diagnosis in technical systems described by linear dynamic models is considered. Behavior of residual generated by observer-based generator realized in a canonical form is analized. Procedure of robust observer design ensuring the best robust property of the residual with respect to disturbances in the form a stationary variate is suggested.
\end{abstract}

Key words: dynamic systems, fault detection, observers, robustness

\section{INTRODUCTION}

There are many papers and books devoted to the problem of robust fault detection and isolation (Low et al, 1986; Patton, 1994; Chen \& Patton, 1999; Blanke et al, 2003). This problem is completely solved in the case when a residual generator has a form of linear parity relations; in the case of observer-based generator there are partial solutions only.

Consider a class of systems described by linear model

$$
x(t+1)=F x(t)+G u(t)+L \rho(t), \quad y(t)=H x(t) .
$$

Here $x \in X, u \in U$, and $y \in Y$ are vectors of state, control, and output; $F, G, L$, and $H$ are constant matrices of appropriate dimensions; $\rho(t)$ is unknown function of time describing disturbances. It is supposed that a residual generator (a diagnostic observer) is described by equations

$$
x_{*}(t+1)=F_{*} x_{*}(t)+G_{*} u(t)+J y(t)+K r(t), \quad y_{*}(t)=H_{*} x_{*}(t)
$$

where $K$ is a feedback gain, $r(t)$ is a residual generated in a form $r(t)=R y(t)-y_{*}(t)$ for some matrix $R$. It is assumed that if there are no faults, the equality $r(t)=0$ holds, $r(t) \neq 0$ otherwise. Thus, the residual gives information whether or not a fault occurs in the system.

To determine the matrices $R, F_{*}, G, J, H_{*}$, and $K$, assume that the matrix $\Phi$ exists such that $x_{*}(t)=\Phi x(t) \forall t$ in the unfaulty case. It is known (Zhirabok, 2010) that matrices describing systems (1) and (2) satisfy the following equations:

$$
\Phi F=F_{*} \Phi+J_{*} H, \quad R H=H_{*} \Phi, \quad G_{*}=\Phi G, \quad \Phi L=0 .
$$

The equation $\Phi L=0$ ensures the invariance of $r(t)$ with respect to the disturbance $\rho(t)$. In practice, as a rule, disturbances are such that $\Phi L \neq 0$ therefore the robust observer must be constructed. In this paper, the algorithm of finding such solution is presented.

\section{RESIDUAL ANALYSIS}

To analyze a demand of robustness, consider an expression for the residual $r(t)$ which can be transformed as follows:

$$
r(t)=R H x(t)-H_{*} x_{*}(t)=H_{*} \Phi x(t)-H_{*} x_{*}(t)=H_{*} e(t),
$$

where $e(t)=\Phi x(t)-x_{*}(t)$. Using models (1), (2), and (3), one obtains an equation for the error $e(t)$ :

$$
e(t+1)=\Phi x(t+1)-x_{*}(t+1)=\left(F_{*}-K H_{*}\right) e(t)+\Phi L \rho(t) .
$$

Consider the case when $\Phi L \neq 0$ and suppose that the disturbance $\rho(t)$ is a stationary variate with the mean $\bar{\rho}$. Supposing that eigenvalues of the matrix $F_{*}-K H_{*}$ are less than 1 modulo, one obtains from (5) an equation for the steadystate mean $\bar{e}$ of the error $e$ :

$$
\bar{e}=\left(F_{*}-K H_{*}\right) \bar{e}+\Phi L \bar{\rho}
$$

Suppose that a pair $\left(\left(F_{*}-K H_{*}\right), H_{*}\right)$ is observable and consider for simplicity $k=3$ where $k$ is a dimension of the observer. Observability of $\left(\left(F_{*}-K H_{*}\right), H_{*}\right)$ means that the system described by equations (4) and (5) with the state vector $e(t)$ and the output vector $r(t)$ can be transformed via nonsingular matrix $T$ into the identification canonical form

$$
T\left(F_{*}-K H_{*}\right) T^{-1}=\left(\begin{array}{ccc}
-k_{1} & 1 & 0 \\
-k_{2} & 0 & 1 \\
-k_{3} & 0 & 0
\end{array}\right), \quad H_{*} T^{-1}=\left(\begin{array}{lll}
1 & 0 & 0
\end{array}\right)
$$

where $k_{1}, k_{2}, k_{3}$ are feedback coefficients. It is known that the matrix $T\left(F_{*}-K H_{*}\right) T^{-1}$ has the same eigenvalues $\lambda_{1}, \lambda_{2}$, and $\lambda_{3}$ as the one $F_{*}-K H_{*}$ because they are similar matrices. One can obtain from (6) after simple transformations:

$$
T \bar{e}=T\left(F_{*}-K H_{*}\right) T^{-1} \overline{T e}+T \Phi L \bar{\rho}=\left(\begin{array}{ccc}
-k_{1} & 1 & 0 \\
-k_{2} & 0 & 1 \\
-k_{3} & 0 & 0
\end{array}\right) T \bar{e}+T \Phi L \bar{\rho}
$$

Denoting $\bar{e}_{k}=T e$ and taking in account the relation $\bar{r}=H_{*} \bar{e}=H_{*} T^{-1} \overline{T e}$, one obtains for the steady-state means $\bar{e}_{k}$ and $\bar{r}$ :

$$
\left(\begin{array}{ccc}
k_{1}+1 & -1 & 0 \\
k_{2} & 1 & -1 \\
k_{3} & 0 & 1
\end{array}\right) \bar{e}_{k}=T\left(\begin{array}{c}
\Phi_{1} L \bar{\rho} \\
\Phi_{2} L \bar{\rho} \\
\Phi_{3} L \bar{\rho}
\end{array}\right)=\left(\begin{array}{c}
\alpha_{1} \\
\alpha_{2} \\
\alpha_{3}
\end{array}\right), \quad \bar{r}=\left(\begin{array}{lll}
1 & 0 & 0
\end{array}\right) \bar{e}_{k} .
$$

Summing over all rows of matrices in (7), one obtaines the equality $\left(k_{1}+k_{2}+k_{3}+1 \quad 0 \quad 0\right) \bar{e}_{k}=\alpha_{1}+\alpha_{2}+\alpha_{3}$; because $\bar{r}=\left(\begin{array}{lll}1 & 0 & 0\end{array}\right) \bar{e}_{k}$, then 


$$
\bar{r}=\left(\alpha_{1}+\alpha_{2}+\alpha_{3}\right) /\left(k_{1}+k_{2}+k_{3}+1\right) .
$$

Since the coefficients $k_{1}, k_{2}, k_{3}$ and eigenvalues satisfy the relations $k_{1}=-\left(\lambda_{1}+\lambda_{2}+\lambda_{3}\right), k_{2}=\lambda_{1} \lambda_{2}+\lambda_{1} \lambda_{3}+\lambda_{2} \lambda_{3}$, and $k_{3}=-\lambda_{1} \lambda_{2} \lambda_{3}$, then $k_{1}+k_{2}+k_{3}+1=\left(1-\lambda_{1}\right)\left(1-\lambda_{2}\right)\left(1-\lambda_{3}\right)$.

It can be shown that a structure of (7) and (8) remains for arbitrary $k$ therefore in the common case (8) has a form

$$
\bar{r}=\sum_{i=1}^{k} \alpha_{i} / \prod_{i=1}^{k}\left(1-\lambda_{i}\right)
$$

Taking into account (7), one can rewrite the sum $\sum_{i=1}^{k} \alpha_{i}$ in (9) in a form $T \sum_{i=1}^{k} \Phi_{i} L \bar{\rho}$. As a rule, the mean $\bar{\rho}$ of the disturbance is unknown, then to minimize the mean of the residual $\bar{r}$, it is necessary to minimize the sum $T \sum_{i=1}^{k} \Phi_{i} L$.

\section{ROBUS OBSERVER DESIGN}

It is known (Patton, 1994; Zhirabok, 2010) that the observer can be built in the canonical form with matrices

$$
F_{*}=\left(\begin{array}{ccccc}
0 & 1 & 0 & \cdots & 0 \\
0 & 0 & 1 & \cdots & 0 \\
\vdots & \vdots & \vdots & \ddots & \vdots \\
0 & 0 & 0 & \cdots & 0
\end{array}\right), \quad H_{*}=\left(\begin{array}{lllll}
1 & 0 & 0 & \cdots & 0
\end{array}\right)
$$

In this case one can set $T=I_{k \times k}$, where $I_{k \times k}$ is a unit matrix and the sum $T \sum_{i=1}^{k} \Phi_{i} L$ takes a form $\sum_{i=1}^{k} \Phi_{i} L$.

It can be shown that for canonical form (10) equations in (3) can be rewritten in a form

$$
\begin{array}{r}
\Phi_{1}=R H, \quad \Phi_{i} F=\Phi_{i-1}+J_{i} H, \quad i=1, \ldots, k-1, \\
\Phi_{k} F=J_{k} H, \quad(11)
\end{array}
$$

where $\Phi_{i}$ and $J_{i}$ are the $i$-th rows of the matrices $\Phi$ and $J$ respectively, $i=1, \ldots, k$. Equations (11) can be transformed into the single one as follows:

$$
R H F^{k}=J_{1} H F^{k-1}+J_{2} F^{k-1}+\ldots+J_{k} H .
$$

To solve the problem of the robust observer design, find the set $\Sigma$ of all linearly independent solutions to this equation for $k=n$ and $R \neq 0$. It can be shown that if some solution to (12) with $k<n$ exists, then it can be obtained as a linear combination of some solutions from $\Sigma$ which gives $J_{k+1}=\ldots=J_{n}=0$. This corresponds to solution with a dimension $k<n$.

$$
\text { If } \quad\left(\begin{array}{llll}
R & -J_{1} & \ldots & -J_{n}
\end{array}\right)=v_{1}\left(R^{(1)}-J_{1}^{(1)} \quad \ldots \quad-J_{n}^{(1)}\right)+
$$
$v_{2}\left(R^{(2)}-J_{1}^{(2)} \quad \ldots-J_{n}^{(2)}\right)$ is a linear combination of two solutions from $\Sigma$, then

$$
\begin{gathered}
\Phi_{1}=R H=v_{1} R^{(1)} H+v_{2} R^{(2)} H=v_{1} \Phi_{1}^{(1)}+v_{2} \Phi_{1}^{(2)}, \\
\Phi_{2}=\Phi_{1} F-J_{1} H=\left(v_{1} \Phi_{1}^{(1)}+v_{2} \Phi_{1}^{(2)}\right) F-\left(v_{1} J_{1}^{(1)}+v_{2} J_{1}^{(2)}\right) H= \\
v_{1}\left(\Phi_{1}^{(1)} F-J_{1}^{(1)} H\right)+v_{2}\left(\Phi_{1}^{(2)} F-J_{1}^{(2)} H\right)=v_{1} \Phi_{2}^{(1)}+v_{2} \Phi_{2}^{(2)}, \ldots
\end{gathered}
$$

This means that rows of the matrix $\Phi$ can be obtained as a linear combination of the matrices $\Phi^{(1)}$ and $\Phi^{(2)}$ rows.

$$
\text { Denote } \Phi_{i}^{\Sigma}=\left(\begin{array}{c}
\Phi_{i}^{(1)} \\
\vdots \\
\Phi_{i}^{(N)}
\end{array}\right), \quad i=1, \ldots, n, \quad \Phi^{\Sigma}=\left(\begin{array}{c}
\sum_{i=1}^{n} \Phi_{i}^{(1)} \\
\vdots \\
\sum_{i=1}^{n} \Phi_{i}^{(N)}
\end{array}\right) \text {, }
$$

$N=|\Sigma|$. Suppose that $v=\left(\begin{array}{lll}v_{1} & \ldots & v_{N}\end{array}\right)$ is a row vector of weight coefficients and $\Phi_{i}=\left(\begin{array}{lll}v_{1} & \ldots & v_{N}\end{array}\right) \Phi_{i}^{\Sigma}, \quad i=1, \ldots, n$. Then $\sum_{i=1}^{n} \Phi_{i}=\left(\begin{array}{lll}v_{1} & \ldots & v_{N}\end{array}\right) \sum_{i=1}^{n} \Phi_{i}^{\Sigma}$. It follows from above that $\sum_{i=1}^{n} \Phi_{i}=\left(\begin{array}{lll}v_{1} & \ldots & v_{N}\end{array}\right) \Phi^{\Sigma}$.

The best choise for the vector $v=\left(\begin{array}{llll}v_{1} & \ldots & v_{N}\end{array}\right)$ can be obtained on the basis of singular value decomposition of the matrix $\Phi^{\Sigma}$ (Low et al, 1986): $\Phi^{\Sigma}=U_{\Phi} \cdot \Sigma_{\Phi} \cdot V_{\Phi}$, where $U_{\Phi}$ and $V_{\Phi}$ are orthogonal matrices, $\Sigma_{\Phi}=\left(\begin{array}{c}\operatorname{diag}\left(\sigma_{1}, \ldots, \sigma_{n}\right) \\ 0\end{array}\right)$, $0 \leq \sigma_{1} \leq \sigma_{2} \leq \ldots \leq \sigma_{n}$ are singular values of $\Phi^{\Sigma}$. It is known that the best choise for $v=\left(\begin{array}{lll}v_{1} & \ldots & v_{N}\end{array}\right)$ is the first transposed column of $U_{\Phi}$.

Algorithm

1. Find all linearly independent solutions to (12) in a form of the set $\Sigma$ of rows $\left(\begin{array}{llll}R^{(j)} & -J_{1}^{(j)} & \ldots & -J_{n}^{(j)}\end{array}\right), j=1, \ldots, N$.

2. Find a set of rows $\Phi_{i}^{(j)}$ of matrix $\Phi^{(j)}$ on the basis of (11), $j=1, \ldots, N, i=1, \ldots, n$.

3. Construct the matrix $\Phi^{\Sigma}$ and find its singular value decomposition. Obtain the vector $v=\left(\begin{array}{llll}v_{1} & \ldots & v_{N}\end{array}\right)$ and rows of the matrices $\Phi, J$, and $R$ respectively: $\Phi_{i}=v \Phi_{i}^{\Sigma}$, $J_{i}=v\left(\begin{array}{c}J_{i}^{(1)} \\ \vdots \\ J_{i}^{(N)}\end{array}\right), i=1, \ldots, n, R=v\left(\begin{array}{c}R^{(1)} \\ \vdots \\ R^{(N)}\end{array}\right)$; set $G_{*}=\Phi G$.

The observer built according to this algorithm has the best robust property but its dimension can exceed over the minimal possible dimension of the diagnostic observer with the same other properties.

\section{CONCLUSION}

The paper presents the robust observer design procedure ensuring the best robust property of the residual with respect to disturbances. This procedure can be extended on the nonlinear case when the logic-dynamic approach developed in (Zhirabok, 2010) is used for the observer design.

\section{REFERENCES}

Blanke, M.; Kinnaert, M.; Lunze, J. \& Staroswieki, M. (2003). Diagnosis and Fault Tolerant Control, Springer-Verlag, ISBN 978-3-540-35652-3, New York

Chen, J. \& Patton R. (1999). Robust model-based fault diagnosis, Kluwer Academic Publishers, ISBN 0792384113, Boston

Lou, X.; Willsky, A. \& Verghese, G. (1986). Optimally robust redundancy relations for failure detection in uncertain systems. Automatica, Vol. 22, pp. 333-344, ISSN 00051098

Patton, R. (1994). Robust model-based fault diagnosis: the state of the art, Proc. IFAC Sympos. SAFEPROCESS'94, Finland, Espoo, 1994, pp. 1-24

Zhirabok, A.; Kucher, D. \& Filaretov, V. (2010). Achieving robustness at diagnosis of nonlinear systems, Automation and Remote Control, Vol. 71, pp. 142-155, ISSN 00051179 